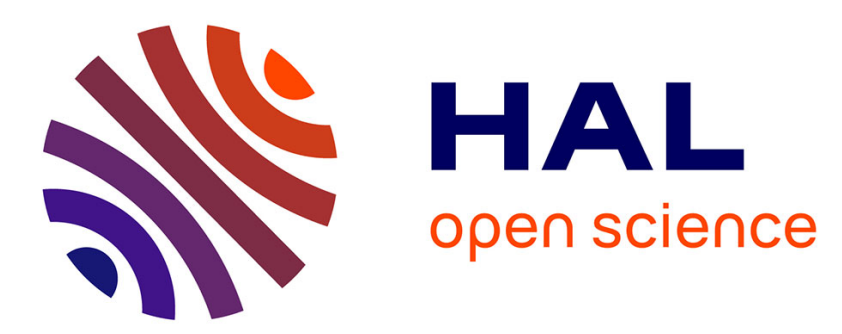

\title{
An Enhanced Structure-from-Motion Paradigm based on the Absolute Dual Quadric and Images of Circular Points
}

\author{
Lilian Calvet, Pierre Gurdjos
}

\section{- To cite this version:}

Lilian Calvet, Pierre Gurdjos. An Enhanced Structure-from-Motion Paradigm based on the Absolute Dual Quadric and Images of Circular Points. International Conference on Computer Vision - ICCV 2013, Dec 2013, Sydney, Australia. pp. 985-992. hal-01166965

\section{HAL Id: hal-01166965 \\ https://hal.science/hal-01166965}

Submitted on 23 Jun 2015

HAL is a multi-disciplinary open access archive for the deposit and dissemination of scientific research documents, whether they are published or not. The documents may come from teaching and research institutions in France or abroad, or from public or private research centers.
L'archive ouverte pluridisciplinaire HAL, est destinée au dépôt et à la diffusion de documents scientifiques de niveau recherche, publiés ou non, émanant des établissements d'enseignement et de recherche français ou étrangers, des laboratoires publics ou privés. 


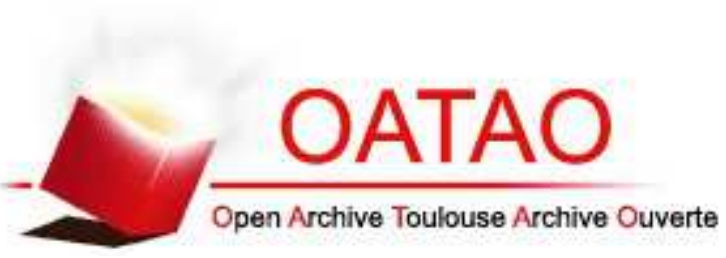

\section{Open Archive TOULOUSE Archive Ouverte (OATAO)}

OATAO is an open access repository that collects the work of Toulouse researchers and makes it freely available over the web where possible.

This is an author-deposited version published in : http://oatao.univ-toulouse.fr/ Eprints ID : 12849

To link to this article : DOI: $\underline{10.1109 / I C C V .2013 .126}$

URL : http://dx.doi.org/10.1109/ICCV.2013.126

To cite this version : Calvet, Lilian and Gurdjos, Pierre An Enhanced Structure-from-Motion Paradigm based on the Absolute Dual Quadric and Images of Circular Points. (2013) In: International Conference on Computer Vision - ICCV, 2013, 1 December 2013 - 8 December 2013 (Sydney, Australia).

Any correspondance concerning this service should be sent to the repository administrator: staff-oatao@ listes-diff.inp-toulouse.fr 


\section{An Enhanced Structure-from-Motion Paradigm based on the Absolute Dual Quadric and Images of Circular Points}

\author{
Lilian Calvet \\ IRIT-ENSEEIHT, Toulouse, France \\ lilian.calvet@enseeiht.fr
}

\author{
Pierre Gurdjos \\ IRIT-ENSEEIHT, Toulouse, France \\ pgurdjos@enseeiht.fr
}

\begin{abstract}
This work aims at introducing a new unified Structurefrom-Motion (SfM) paradigm in which images of circular point-pairs can be combined with images of natural points. An imaged circular point-pair encodes the $2 D$ Euclidean structure of a world plane and can easily be derived from the image of a planar shape, especially those including circles. A classical SfM method generally runs two steps: first a projective factorization of all matched image points (into projective cameras and points) and second a camera selfcalibration that updates the obtained world from projective to Euclidean. This work shows how to introduce images of circular points in these two SfM steps while its key contribution is to provide the theoretical foundations for combining "classical" linear self-calibration constraints with additional ones derived from such images. We show that the two proposed SfM steps clearly contribute to better results than the classical approach. We validate our contributions on synthetic and real images.
\end{abstract}

\section{Introduction}

A now classical Structure-from-Motion (SfM) paradigm basically consists of two key steps: (i) compute a projective reconstruction of cameras and points; (ii) update the obtained projective representation to Euclidean by locating the absolute conic on the plane at infinity [3]. One key issue in SfM is to solve $(i)$ by simultaneously using all the matched features in all images i.e., all the constraints ensuring the scene rigidness. This is exactly what factorizationbased paradigms aim at. They rely on the factorization of a measurement matrix consisting of matched points scaled to their projective depths, into two lower-rank matrices, one representing the camera motion and one representing the 3D points of the scene structure, both w.r.t. some unknown projective world representation. A method that solves (ii) is socalled self-calibration and is always achieved under some assumptions on the camera parameters [10] as the absolute conic refers to the (unique) imaginary circle at infinity in projective 3-space which is fixed under general motions of the camera. Once the absolute conic is recovered, a projective transfomation can be applied to the world space to update it to Euclidean.

We focus here on direct approaches which rely on the fact that the absolute conic may be represented by a single equation in dual projective space (and hence by a single matrix) as the assemblage of its tangent planes i.e., as the so-called absolute dual quadric. The absolute dual quadric and its use in computer vision has been introduced by Triggs in 1997 [13] but surely popularised in 1999 by the linear least-squares algebraic solution of Pollefeys et al. $[5,6,10]$. In this approach, it is assumed the hypothesis $(\mathcal{H})$ of a camera with known intrinsics except a time-varying focal length. A minimal set of 3 cameras is required to obtain a linear least squares solution for the $4 \times 4$ symmetric matrix $Q_{\infty}^{*}$ of the dual absolute quadric, if one omits that $Q_{\infty}^{*}$ is rank-3 and semidefinite positive. It is known [3, p. 468] that any method that enforces such constraints a posteriori suffers critical motions if not explicitly dealt with [2].

Problem statement and contributions. In this work, it is assumed to have at one's disposal a set of views where some "natural" points of interest are matched and the 2D Euclidean structure of a world plane is available (e.g., as the plane-to-image homography), but possibly not in all views. Fig. 1(a)(b) gives two examples of such view sets. On the one hand, omitting the available 2D Euclidean structure, if we run the classical SfM approach $[5,6,10]$ with these sets as input, it fails because either there are too few views or matched points or the camera motion is critical [2]. On the other hand, if we run a planar-based camera calibration approach for 3D reconstruction, e.g. [16], it also fails because the pattern is too small and/or not seen in all views. This works aims at introducing a new unified SfM paradigm, getting the best out of the two SfM and plane-based reconstruction approaches, and overcoming their disadvantages, without requiring off-line calibration. In our examples, using the new paradigm, we were able to obtain a satisfactory final dense reconstruction, which was provided by PMVS 

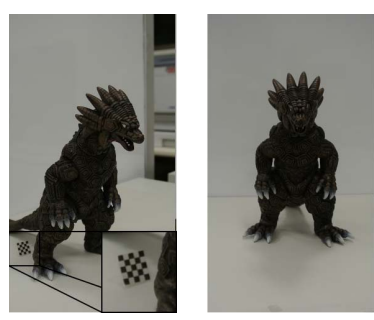

(a)
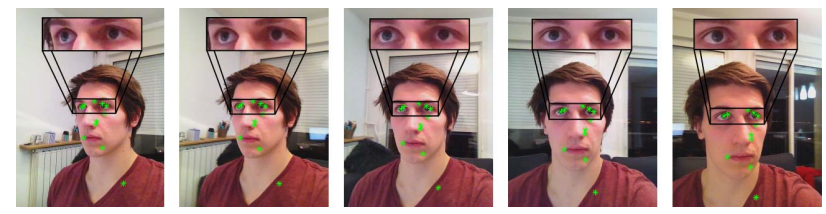

(b)

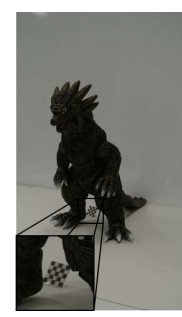

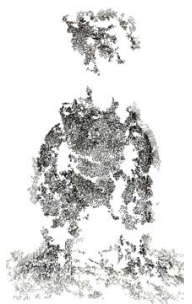
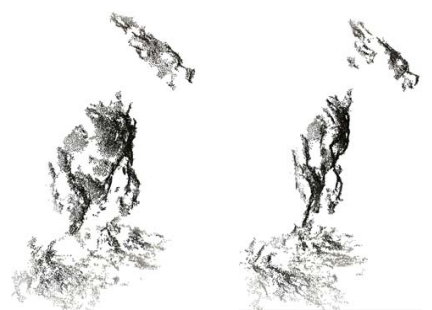

(c)
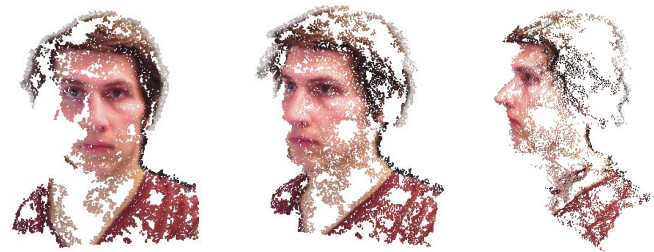

(d)

Figure 1: (a) Three views of a dragon where 54 points can be matched and a very small checkerboard-style grid is visible except in view 2 . The $2 \mathrm{D}$ Euclidean structure is computed from the plane-to-image homography induced by the grid (no other points were used). (b) Five views of a human face where 11 points can be matched and the 2D Euclidean structure is given by the imaged circular points which are two (complex) intersection points of the two coplanar circles formed by the eye's iris outlines. (c) No consistent Euclidean reconstruction was obtained using either Bouguet's Toolbox or POLLEFEYs' method $[5,6,10]$. (c)+(d) Only the proposed method, starting from the same projective reconstruction than other methods, by adding circular points in self-calibration (see Table 4), was able to provide PMVS [1] — even if the planar shape was very small— accurate Euclidean cameras. This yielded the dense reconstruction seen here from three different viewpoints.

[1] from the obtained Euclidean cameras along with the set of views, as seen in Fig. 1(c)(d).

In this work, we treat the circular point-pair (CP-pair) of a plane as a feature. The CP-pair consists of two complex conjugate points at infinity [3] whose images, easily derived from the geometry of any planar shape like $[15,16,11]$, define the well-known plane-based calibration constraints [16]. Our key idea is that incorporating the images of CPpairs into the SfM algorithm sequence, namely $(i)$ in projective factorization scheme mixing images of natural points and images of CP as well and (ii) in the Euclidean update of the obtained projective reconstruction. The contribution is double: the first one is to show how to determine the complex projective depths of the images of $\mathrm{CP}$ and how to we deal with the subproblem of missing data i.e., when the $\mathrm{CP}$ are not seen in all views. The key contribution is secondly to describe how the "classical" Pollefey's self-calibration constraints can be combined with additional ones derived from the images of the CP. In fact, we give the theoretical foundation proving that both Pollefey's constraints and the proposed ones define 3D lines through the camera centre that cut the absolute conic i.e., that are tangent-lines of the absolute conic. Since the Absolute Quadratic Complex is the assemblage of these tangent -lines, as a result, surprisingly enough, this means that the self-calibration methods of Pollefeys et al. but also [5] Ponce et al. [7], Valdés et al. [14] basically rely on a same paradigm.

Paper organisation. At the end of this section, some background on projective geometry in 3-space is reminded. In section 2, we show how to introduce images of $\mathrm{CP}$ in the projective factorization algorithm. In section 3 , we describe how the "classical" self-calibration constraints can be combined with additional ones derived from the images of the CP.

Background. Any projective plane intersects the absolute conic $\Omega_{\infty}$ at a (complex) circular point-pair. Planes that are parallel share the same circular point-pair which is also the common point-pair of all circles lying on such parallel planes [3, p. 52-53]. The image of a circular point only have 4 dof as its (complex) homogeneous 3 -vector is defined up to a complex factor. Throughout this paper $\mathbf{I}_{ \pm}$ will represent the image of a circular-point pair and we will assume that it is part of the input data, but possibly not for all the views. When it is not necessary to distinguish betwen $\mathbf{I}_{+}$and $\mathbf{I}_{-}$, the image of a circular point will simply be referred to as $\mathbf{I}$.

\section{Projective factorization with circular points}

The statement of the projective factorization problem assumes that $M$ world points are viewed by $N$ different cameras and that the $M N$ image points are gathered within a measurement $3 N \times M$-matrix. The first key issue is that of rescaling all image points such that, in absence of measurement noise, the measurement matrix is ensured to have rank 4. The second key issue is that of dealing with missing data i.e., when world points are not seen in all views. In 
our case, we assume the image points to be projections of "natural" points and circular points. Our contribution is to solve the two issues by describing linear solutions. While the proposed method can deal with more than one circular point, we will focus on the case of a single circular point which is the only case that theoretically requires images of natural points in addition. The input data consists of $(a)$ a sequence of view indices $\left(j_{0}, \ldots, j_{p}, j_{c}, \ldots, j_{N}\right)$ in the processing order (which can be arbitrary) ; (b) the set of unscaled images $\left\{\mathbf{I}_{j}\right\}$ of circular points and, for each view, the set of (already) rescaled images $\left\{\tilde{\mathbf{u}}_{k}\right\}$ of natural point $k(1 \leq k \leq P)$; (c) inter-view epipoles (represented by vector $\mathbf{e}_{j_{p} j_{c}}$ in view $j_{c}$ imaging the $j_{p}$-th camera centre). Regarding $(b)$, any rescaling method can be used e.g., that in $[4,12][3$, p. 444].

Rescaling circular-point images. Rescaling the image $\mathbf{I}_{j}$ of a circular point is not straightforward as it must be multiplied by a complex factor $\mu_{j}$. We propose an iterative algorithm (see table 1) that rescales the available images of circular points in such a way that, at the current step processing view $j_{c}$, the images have already been rescaled in previous view $j_{p}$. Initially, the images in the first view $j_{0}$ are arbitrarily scaled i.e., $\mu_{j_{0}}=1$.

We first write $\mu_{j}=a_{j}+i b_{j}$, with $a_{j}, b_{j} \in \mathbb{R}$, such that

$$
\mu_{j} \mathbf{I}_{j}=\mathrm{C}_{j}\left[\begin{array}{cc}
a_{j} & b_{j} \\
-b j & a_{j}
\end{array}\right]\left(\begin{array}{l}
1 \\
i
\end{array}\right)
$$

where $i^{2}=-1$ and $\mathrm{C}_{j}$ denotes the $3 \times 2$-matrix whose columns stack the real and imaginary 3 -vectors of $\mathbf{I}_{j}$. Our solution relies on two linear equations. The first one is

$$
\mathrm{C}_{j_{c}}\left[\begin{array}{cc}
a_{j_{c}} & b_{j_{c}} \\
-b j_{c} & a_{j_{c}}
\end{array}\right]-\mu_{j_{p}} \mathrm{H}_{j_{p} j_{c}} \mathrm{C}_{j_{p}}=\mathbf{0}_{3 \times 2}
$$

that is a rewriting of $\mu_{j_{c}} \mathbf{I}_{j_{c}}-\mu_{j_{p}} \mathrm{H}_{j_{p} j_{c}} \mathbf{I}_{j_{c}}=0$ which transfers the image of a circular point from previous view $j_{p}$ to current view $j_{c}$, via the homography $\mathrm{H}_{j_{p} j_{c}}$, induced by some world plane that contains the circular point-pair. Given the matched rescaled images $\left(\tilde{\mathbf{u}}_{j_{p}}, \tilde{\mathbf{u}}_{j_{c}}\right)$ of a natural world point, the second one is

$$
\left[\mathbf{e}_{j_{p} j_{c}}\right]_{\times}\left(\mathrm{H}_{j_{p} j_{c}} \tilde{\mathbf{u}}_{j_{p}}-\tilde{\mathbf{u}}_{j_{c}}\right)=\mathbf{0}_{3}
$$

where $\mathbf{e}_{j_{p} j_{c}}$ is the epipole in view $j_{c}$ imaging the $j_{p}$-th camera centre and $\tilde{\mathbf{u}}_{j}$ is the rescaled image point in view $j$. This is basically a direct rewriting of the standard epipolar constraint $\mathrm{F}_{j_{p} j_{c}} \tilde{\mathbf{u}}_{j_{p}}-\left[\mathbf{e}_{j_{p} j_{c}}\right]_{\times} \tilde{\mathbf{u}}_{j_{c}}=\mathbf{0}_{3}$, which involves the fundamental matrix $\mathrm{F}_{j_{p} j_{c}}=\left[\mathbf{e}_{j_{p} j_{c}}\right]_{\times} \mathrm{H}_{j_{p} j_{c}}$ mappings a point in view $j_{p}$ to its epipolar line in view $j_{c}$.

Keeping in mind that, $\mu_{j_{p}}$ has been estimated in previous step $j_{p}$, clearly (1) is an homogeneous equation that linearly constrains the unknown scalars $a_{j_{c}}, b_{j_{c}}$ (which rescaled $\tilde{\mathbf{I}}_{j_{c}}$ ) plus the unknowns in $\mathrm{H}_{j_{p} j_{c}}$. On the other hand, (2) is an inhomogeneous equation which is linear in $\mathrm{H}_{j_{p} j_{c}}$. It is worthy of mention that, using only one CP-pair, there is a 1-dof ambiguity in $\mathrm{H}_{j_{p} j_{c}}$ in both equations (1) and (2). This is due to the fact that an absolute point-pair is common to a 1D set of parallel planes: if we represent their (common) vanishing lines in view $j_{p}$ by the 3 -vectors $\mathbf{v}_{j_{p}}$, then the set induces a family of inter-view homographies of the form $\mathrm{H}(z)=\mathrm{H}_{j_{p} j_{c}}-z \mathbf{e}_{j_{p} j_{c}} \mathbf{v}_{j_{p}}^{\top}$. In other words, we can replace, in (1) and (2), the homography $\mathrm{H}_{\infty}^{k j}$ by any $\mathrm{H}(z)$ and observe that (1) and (2) still hold. To fix this dof, we assume that $z$ is such that two elements in $\mathrm{H}_{\infty}^{k j}$ are equal (clearly such $z$ exists). As a result, $\mathrm{H}_{\infty}^{k j}$ is parameterised by 8 scalars instead of 9 . As a counting argument, it can be seen that the configuration of the image of one circular point and the (scaled) matched images of $P$ natural points introduces 10 unknowns while providing $2 P+6$ equations. Hence, $P=2$ natural points is a minimal theoretical data set ${ }^{1}$. An overdetermined set of equations can be easily solved in a linear least squares manner. Computationally-wise, the complete iterative algorithm is given in Table 1.

1) Select a first view $j_{0}$ and let $\mu_{j_{0}}=1$

2) Set $j_{p} \leftarrow j_{0}$ and select a current view $j_{c}$

3) If the circular point is visible in in views $j_{p}$ and $j_{c}$ then

4) Find $\mathcal{S}$, the set of natural points visible in views $j_{p}$ and $j_{c}$ 5) Using all $p \in \mathcal{S}$, rewrite $(1,2)$ as a linear system $\mathbf{A X} \approx \mathbf{b}$ where $\mathbf{X} \in \mathbb{R}^{10}$ stacks the 8 elements of $\mathrm{H}_{j_{p} j_{c}}$ and $a_{j_{c}}, b_{j_{c}}$

6) Solve $\min _{\mathbf{X}}\|\mathbf{A} \mathbf{X}-\mathbf{b}\|_{F}^{2}$

7) Extract $\mu_{j_{c}}=a_{j_{c}}+i b_{j_{c}}$ from the solution $\hat{\mathbf{X}}$

9) end

8) Set $\tilde{\mathbf{I}}_{j_{c}} \leftarrow \mu_{j_{c}} \tilde{\mathbf{I}}_{j_{c}}$

10) Set $j_{p} \leftarrow j_{c}$, select a new view $j_{c}$ and go to 2 ).

Table 1: Algorithm scaling the images of circular points

Missing data. Suppose that we ran the algorithm of Table 2 only for the visible images of circular points. Hence, some (block) entries of the measurement matrix are missing. Thanks to equations (1) and (2), we are able to predict these missing data. Assume that the circular point is not seen in view $j_{c}$ but is viewed in $V$ other views, of which view $j_{p}$ is taken. A linear solution exists based on two equations involving the matched rescaled images $\left(\tilde{\mathbf{u}}_{j_{p}}, \tilde{\mathbf{u}}_{j_{c}}\right)$, one of which being a direct rewriting of (1)

$$
\mathrm{D}_{j_{c}}-\mu_{j_{p}} \mathrm{H}_{j_{p} j_{c}} \mathrm{C}_{j_{p}}=\mathbf{0}_{3 \times 2}
$$

and the other one being (2). Now, in addition to $a_{j_{c}}, b_{j_{c}}$ and the 8 elements of $\mathrm{H}_{j_{p} j_{c}}$, the 6 elements of $3 \times 2$-matrix $\mathrm{D}_{j_{c}}$ are also considered as unknowns. As a counting argument, it can be seen that a data set, consisting of the (rescaled) images in $V$ views of the circular point and $P$ natural points, introduces $8 \mathrm{~V}+6$ unknowns while providing $6 \mathrm{~V}+2 \mathrm{PV}$ equations. Hence, $P=3$ if $V=2$ and $P=2$ if $V \geq 3$ is a minimal data set of natural points. Again, an overdetermined set of equations can be easily solved by linear

\footnotetext{
${ }^{1}$ Albeit at least 7 points will be always required toto compute the required fundamental matrix.
} 
least squares optimization. Notice that another way to compute scaled images of circular points in missing views may also be achieved by first computing them, up to a complex scale factor, based on their visible images (at least two) and on fundamental matrices and next, rescaling them as described above. However, this method presents poor results with regard to the one proposed above.

\section{Self-calibration with circular points}

\subsection{Linear self-calibration constraints on $Q_{\infty}^{*}[5,10]$}

We consider a set of $n$ projective cameras, represented by $n$ projection $3 \times 4$-matrices, mapping $3 \mathrm{D}$ points in some projective representation to their images and written as

$$
\mathrm{P}^{j}=\left(\begin{array}{l}
\mathbf{a}^{j \top} \\
\mathbf{b}^{j \top} \\
\mathbf{c}^{j \top}
\end{array}\right) \quad \text { with } j=1 \ldots n .
$$

The absolute conic $\Omega_{\infty}$ projects in dual form in view $j$ as

$$
\boldsymbol{\omega}^{* j} \sim \mathrm{P}^{j} \mathrm{Q}_{\infty}^{*} \mathrm{P}^{j \top}
$$

where $\mathrm{Q}_{\infty}^{*}$ is the rank-3 semidefinite $4 \times 4$-matrix of the dual absolute quadric w.r.t. the projective representation and $\omega^{* j}$ the $3 \times 3$-matrix of its image.

We now replace the hypothesis $(\mathcal{H})$ by the equivalent $\left(\mathcal{H}^{\prime}\right)$ of a camera with square pixels whose principal point coincides with the origin of the pixel coordinate system i.e., we assume the calibration matrix $\mathrm{K}^{j}$ to be diagonal. Under hypothesis $\left(\mathcal{H}^{\prime}\right)$, the image of the absolute conic in dual form writes:

$$
\boldsymbol{\omega}^{* j}=\operatorname{diag}\left(\left(f^{j}\right)^{2},\left(f^{j}\right)^{2}, 1\right)
$$

where $f^{j}$ is the focal length of camera $j$.

Considering the projection equation (5), Pollefeys et al. described in their seminal paper [5] a self-calibration algorithm based on the set of four equations

$$
\begin{aligned}
\mathbf{a}^{j \top} \mathbf{Q}_{\infty}^{*} \mathbf{a}^{j}-\mathbf{b}^{j \top} \mathbf{Q}_{\infty}^{*} \mathbf{b}^{j} & =0 & & \mathbf{a}^{j \top} \mathbf{Q}_{\infty}^{*} \mathbf{b}^{j}=0 \\
\mathbf{a}^{j \top} \mathbf{Q}_{\infty}^{*} \mathbf{c}^{j} & =0 & & \mathbf{b}^{j \top} \mathbf{Q}_{\infty}^{*} \mathbf{c}^{j}=0
\end{aligned}
$$

which are linear in the ten elements of $Q_{\infty}^{*}$. Solving those constraints for multiple cameras e.g., by optimizing in a linear least squares manner in presence of noise, forms the basis of the linear self-calibration algorithms [5].

\subsection{Adding linear circular-point constraints on $Q_{\infty}^{*}$}

In this section, the issue is that of writing the independent self-calibration constraints brought by the image $\mathbf{I}_{ \pm}$of a circular point-pair. A trivial investigation of constraints on $\boldsymbol{\omega}^{*}$ is to derive the equation dual to $\mathbf{I}^{\top} \boldsymbol{\omega} \mathbf{I}=0$, where $\boldsymbol{\omega} \sim$ $\left(\boldsymbol{\omega}^{*}\right)^{-1}$ is the image of $\Omega_{\infty}$ in "primal" form. This leads to the (complex) equation:

$$
\mathbf{T}^{\top} \boldsymbol{\omega}^{*} \mathbf{T}=0
$$

where $\mathbf{T}$ is the 3 -vector of the line tangent to $\boldsymbol{\omega}$ at $\mathbf{I}$, defined by to the polar-pole relation $\mathbf{T}=\boldsymbol{\omega} \mathbf{I}$ [3]. Going further is not so straightforward as some issues must be addressed just yet : (i) $\mathbf{T}$ seemingly depends on $\boldsymbol{\omega}$ which is unknown; (ii) if $\mathbf{T}$ was given, it is clear that, by requiring the real and imaginary parts of the complex equation (9) to be zero, it would yield two linearly real dependent equations on $\omega^{*}$ as $\boldsymbol{\omega}^{*}$ depends on the sole parameter $f$. Hence, it does not really make sense to seek additional linear equations on $\omega^{*}$; (iii) as the unknown is $Q_{\infty}^{*}$ related to $\omega^{*}$ by (5), it is required to interpret (9) in 3-space.

All these issues can be solved by introducing the 3D linepair $\mathrm{L}\left(\mathbf{I}_{ \pm}\right)$which back-projects $\mathbf{I}_{ \pm}$and cuts the absolute conic (see fig. 2). Our key contribution is to set up the correct theoretical background in order to answer the question "what are the additional independent equations on $\mathrm{Q}_{\infty}^{*}$ brought by the absolute line-pair $\mathrm{L}\left(\mathbf{I}_{ \pm}\right)$?", emphasing the term "independent".

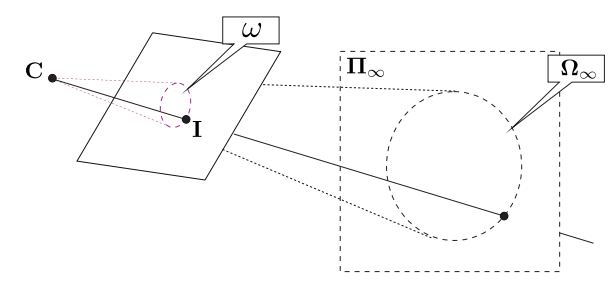

Figure 2: The line through the camera centre $\mathbf{C}$ and the image $\mathbf{I}$ of a circular point touches the absolute conic $\Omega_{\infty}$.

Theoretical background. The following proposition reveals the linear constraints on $\mathrm{Q}_{\infty}^{*}$ brought by line $\mathrm{L}(\mathbf{I})$. Note that a simple way to get this line is by means of its Plücker matrix $\mathrm{L}(\mathbf{I})=\mathrm{P}^{\top}[\mathbf{I}]_{\times} \mathrm{P}$.

Proposition 1 A line $\mathrm{L}$ cuts the absolute conic $\Omega_{\infty}$ iff through $\mathrm{L}$ it passes a plane tangent to $\Omega_{\infty}$, i.e, a (complex) plane $\Pi \in \mathbf{Q}_{\infty}^{*}$, hence satisfying

$$
\Pi^{\top} \mathbf{Q}_{\infty}^{*} \boldsymbol{\Pi}=0,
$$

and a (complex or real) plane $\mathbf{q}$ conjugate to $\boldsymbol{\Pi}$ w.r.t. $\mathbf{Q}_{\infty}^{*}$, i.e, satisfying

$$
\Pi^{\top} \mathbf{Q}_{\infty}^{*} \mathbf{q}=0
$$

Proof. Any 3D line can be defined as the intersection of two planes so let $\mathrm{L}$ be defined by planes $\mathbf{r}$ and $\mathbf{s}$. Through $\mathrm{L}$ it also passes two planes that are tangent to $\Omega_{\infty}$, i.e., two planes of $\mathbf{Q}_{\infty}^{*}$, whose vectors can be written as $\mathbf{t}_{j}=\mathbf{r}+u_{j} \mathbf{S}$ ( $j=1,2$ ) with $u_{1}, u_{2}$ being the solutions for $u$ of the quadratic equation $(\mathbf{r}+u \mathbf{s})^{\top} \mathbf{Q}_{\infty}^{*}(\mathbf{r}+u \mathbf{s})=0$. $(\Rightarrow)$ If $\mathrm{L}$ touches $\Omega_{\infty}$, the two tangent planes coincide which entails 
that $u_{1}=u_{2}$ and the discriminant of the left-hand side vanishes i.e., $\left(\mathbf{r}^{\top} \mathbf{Q}_{\infty}^{*} \mathbf{s}\right)^{2}-\left(\mathbf{r}^{\top} \mathbf{Q}_{\infty}^{*} \mathbf{r}\right)\left(\mathbf{s}^{\top} \mathbf{Q}_{\infty}^{*} \mathbf{s}\right)=0$. Hence, if the tangent plane where L touches $Q_{\infty}^{*}$ is either $\mathbf{r}$ or $\mathbf{s}$, then the other one necessarily is a plane that is conjugate to it w.r.t. $\mathbf{Q}_{\infty}^{*}$. $(\Leftarrow)$ The set of planes conjugate to plane $\Pi$ w.r.t. $\mathbf{Q}_{\infty}^{*}$ is formed by all planes through the pole of $\Pi$ w.r.t. $\mathbf{Q}_{\infty}^{*}$ which is the point where $\Pi$ is tangent to $Q_{\infty}^{*}$, that is a circular point. Hence, the $\boldsymbol{\Pi}$ and $\mathbf{q}$ share a line through this circular point so line $\mathrm{L}$ cuts the absolute conic.

Proposition 1 teaches us the fact that, by cutting the absolute conic, $\mathrm{L}(\mathbf{I})$ brings the two complex constraints $(10,11)$ on $Q_{\infty}^{*}$ i.e., four real linear equations by requiring the real and imaginary parts of $(10,11)$ to be zero. It is worthy of note that constraint (10) is actually the one derived from (9) by substituting $\mathrm{PQ}_{\infty}^{*} \mathrm{P}^{\top}$ for $\boldsymbol{\omega}^{*}$ and $\boldsymbol{\Pi}$ for $\mathrm{P}^{\top} \mathbf{T}$. Thus, $\boldsymbol{\Pi}$ is the plane of $Q_{\infty}^{*}$ through $L(\mathbf{I})$, obtained by back-projecting the line $\mathbf{T}$ tangent to $\boldsymbol{\omega}$ at $\mathbf{I}$.

Proposition 2 Under $\left(\mathcal{H}^{\prime}\right)$, the plane of $\mathrm{Q}_{\infty}^{*}$ through $\mathrm{L}(\mathbf{I})$ is

$$
\mathbf{\Pi}=\mathrm{P}^{\top}\left(\begin{array}{c}
\psi_{1} \psi_{3} \\
\psi_{2} \psi_{3} \\
-\left(\psi_{1}^{2}+\psi_{2}^{2}\right)
\end{array}\right)
$$

where $\mathbf{I}=\left(\psi_{1}, \psi_{2}, \psi_{3}\right)^{\top}$ is the image of the circular point.

Proof. On the one hand, under $\left(\mathcal{H}^{\prime}\right)$, the point at infinity of the line $\mathbf{T}$ tangent to the image of the absolute conic at $\mathbf{I}$ is given by $\mathbf{p}_{\infty}=\mathbf{L}^{\infty} \times \mathbf{T}$, where $\mathbf{L}^{\infty}=(0,0,1)^{\top}$ represents the image plane's line at infinity. It is then easy to show that $\mathbf{p}_{\infty}=\left[\mathbf{L}^{\infty}\right]_{\times} \boldsymbol{\omega} \mathbf{I} \sim\left[\mathbf{L}^{\infty}\right]_{\times} \mathbf{I}$, where $\left[\mathbf{L}^{\infty}\right]_{\times}$is the skew-symmetric matrix related to the cross product as defined in [3, p. 581]. As $\mathbf{T}$ contains both $\mathbf{p}_{\infty}$ and $\mathbf{I}$ we can define $\mathbf{T}=\left(\left[\mathbf{L}^{\infty}\right]_{\times} \mathbf{I}\right) \times \mathbf{I}=\left(\psi_{1} \psi_{3}, \psi_{2} \psi_{3},\left(\psi_{1}^{2}+\psi_{2}^{2}\right)\right)^{\top}$. The fact that $\boldsymbol{\Pi}=\mathrm{P}^{\top} \mathbf{T}$ ends the proof. A degenerate case occurs when $\mathbf{I}=(1, \pm i, 0)^{\top}$ i.e., when the $3 \mathrm{D}$ supporting plane is fronto-parallel.

Proposition 1 also teaches us there is an additional independent constraint on $Q_{\infty}^{*}$ in the form of (11).

Proposition 3 A (real) plane through $\mathrm{L}(\mathbf{I})$, that is conjugate to plane $\boldsymbol{\Pi}=\mathrm{P}^{\top} \mathbf{T}$ w.r.t. $\mathrm{Q}_{\infty}^{*}$ as given in (12), is

$$
\mathbf{q}=\mathrm{P}^{\top}\left(\mathbf{I}_{+} \times \mathbf{I}_{-}\right) \text {. }
$$

This plane through $\mathrm{L}(\mathbf{I})$ includes the circular point-pair and can be obtained by back-projecting the vanishing line.

Note that, in the general case, the four equations brought by constraints $(10,11)$ are linearly independent w.r.t. $Q_{\infty}^{*}$.

\subsection{Unifying the linear self-calibration constraints}

What is the link between the circular-point constraints $(10,11)$ and the "original" linear self-calibration constraints $(7,8)$ ? In the light of the insights given in the previous section, we suggest to revisit $(7,8)$ by first complexifying them to get algebraically equivalent equations (superscripts are omitted)

$$
\begin{array}{r}
(\mathbf{a}+i \mathbf{b})^{\top} \mathbf{Q}_{\infty}^{*}(\mathbf{a}+i \mathbf{b})=0 \\
\mathbf{c}^{\top} \mathbf{Q}_{\infty}^{*}\left(\mathbf{a}^{j}+i \mathbf{b}\right)=0
\end{array}
$$

with $i^{2}=-1$. Equations $(14,15)$ can hence be rewritten as:

$$
\begin{aligned}
& \left(\mathrm{P}^{\top}(1, i, 0)^{\top}\right)^{\top} \mathrm{Q}_{\infty}^{*}\left(\mathrm{P}^{\top}(1, i, 0)^{\top}\right)=0 \\
& \left(\mathrm{P}^{\top}(1, i, 0)^{\top}\right)^{\top} \mathrm{Q}_{\infty}^{*}\left(\mathrm{P}^{\top}(0,0,1)^{\top}\right)=0
\end{aligned}
$$

If one looks now at $(16,17)$, one finds out that Pollefeys' linear self-calibration constraints are nothing else than the circular-point constraints like $(10,11)$ but involving in place of the image $\mathbf{I}_{ \pm}$of some circular point-pairthe circular point-pair of the image plane, whose homogeneous coordinates are $\mathbf{I}_{o}=(1, \pm i, 0)^{\top}$ in any Euclidean representation, and hence under hypothesis $\left(\mathcal{H}^{\prime}\right)$. In this case, constraints $(10,11)$ hold with $\Pi=\mathrm{P}^{\top}(1, i, 0)^{\top}$ and $\mathrm{P}^{\top} \mathbf{q}=\mathrm{P}^{\top}(0,0,1)^{\top}$ respectively represent the line tangent to $\boldsymbol{\omega}$ at $\mathbf{I}_{o}$ and the image plane's line at infinity.

The self-calibration equations can now be interpreted: giving projective matrices under hypothesis $(\mathcal{H})$, they express fact that the lines, obtained by back-projecting images of circular points (including the image plane's circular point-pair), cut the absolute conic. It is exactly the same paradigm as in the approaches of Ponce et al. [7, 9] who introduced the "absolute quadratic complex" approach for camera self-calibration, except that these latter also work for a camera with square pixels (and unknown principal point). The problem was naturally formulated in the projective 5-space-i.e., the space of lines- but requires sophisticated fitting algorithms.

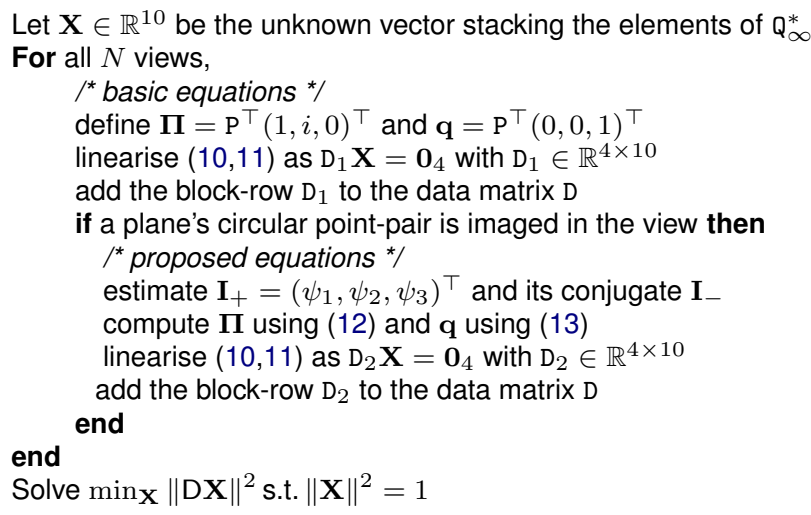

Table 2: Unified self-calibration algorithm based on circular points

\section{Experiments}

We conducted a large number of experiments with synthetic and real images to quantify the performance of the proposed SfM paradigm, using a small amount of natural 
points as input and depending on the presence (or not) of one circular point-pair ( $\mathrm{CP}$ in the sequel). Basically, four use cases were considered: $(i)$ the incorporation (or not) of a CP into the projective factorization algorithm and (ii) the incorporation (or not) of the constraints provided by a $\mathrm{CP}$ in the self-calibration algorithm. We put special emphasis on the interest of projective factorization based on circular points as it offers a twofold advantage. This important point were confirmed by our experiments: more accurate projective cameras were obtained and the reprojections of the obtained projective points yielded "corrected" — and more accurate - image points. .

In the experiments, some algorithms have been provided by the so-called Vincent's SfM Matlab Toolbox [8].

\subsection{Synthetic data}

The performance of the proposed SfM paradigm was assessed by analyzing the accuracy improvement in the final Euclidean reconstruction, computed as the 3D RMS error. Multiple SfM processing sequences were carried out. They are referred to in a generic way as $\mathrm{PF}\left[n_{1}\right]+\mathrm{SC}\left[n_{2}\right]$, which means that we ran a Projective Factorization incorporating the images of $n_{1} \mathrm{CP}$ followed by the Self-Calibration incorporating the constraints provided by $n_{2} \mathrm{CP}$, with $n_{i}=0,1$. Notice that $\mathrm{PF}[0]+\mathrm{SC}[0]$ refers to "classical" $\mathrm{SfM}$ i.e., when only natural points are used. When $n_{2} \geq n_{1}>0$, the images of $\mathrm{CP}$ used in self-calibration were the reprojections of projective $\mathrm{CP}$ computed in the factorization. Experiments were conducted with respect to several variables: the number of views, the number of natural points, the amount of measurement noise and the amount of missing data.

We considered a scene consisting at most of $p \leq 20$ natural points and 0 or $1 \mathrm{CP}$. The $\mathrm{CP}$ were computed as the common projective point-pair of two concentric circles (see [15]). The natural points were randomly distributed in the unit sphere (of diameter $d=2$ units) centered at the origin. Each circle was centered at $[ \pm 0.2, \pm 0.2, z]$ (with $z$ randomly taken in $[-0.2,0.2]$ ) with radius $0.1 d$ while the normal to the supporting plane were randomly distributed in the cone of axis $[0,0,1]$ and angle $\pi / 3$. The cameras were roughly oriented toward the origin, randomly placed at a distance in $[2.85,3.15]$ from the origin. The image resolution is $512 \times 512$ and the focal lengths were randomly chosen in $[850,1150]$. The principal point was assumed to (roughly) coincide with the origin but its pixel coordinates were randomly taken in $[-15,15]^{2}$. A zero-mean Gaussian noise with standard deviation $\sigma$ was added to the image points, including those of the images of the circles from which the images of the $\mathrm{CP}$ were computed.

All the following experiments compared the 3D RMS errors obtained by different algorithm sequences (displayed as legends in Fig. 3) w.r.t. several variables. In Fig. 3(a), 6 views were considered and we let vary the amount of noise disturbing the image point coordinates. In the following experiments, the image points coordinates were always corrupted by a noise of $\sigma=1.0$ pixel. In Fig. 3(b), we let vary the number of views. In Fig. 3(c), 6 views were considered and we let vary the number of points from 6 to 20. In Fig. 3(d), we also considered 6 views but let vary the number of views in which the $\mathrm{CP}$ is visible. The key result is that, compared to "classical SfM" $\mathrm{PF}[0]+\mathrm{SC}[0]$, the accuracy was improved by using either $\mathrm{PF}[0]+\mathrm{SC}[1]$ or $\mathrm{PF}[1]+\mathrm{SC}[0]$ and best by using PF[1]+SC[1]. This means that both proposed algorithms for projective factorisation and self-calibration independently contributed to better results even if the benefits provided by additional constraints from CP images were generally more significant in self-calibration than in projective factorization, particularly when the number of views decreased and the noise increased. Of course, for a very small number of natural points, adding images of $\mathrm{CP}$ in the projective factorization significantly reduced the $3 \mathrm{D}$ RMS error and, in that case, the overall improvement for $\mathrm{PF}[1]+\mathrm{SC}[1]$ was mostly due to the factorization. All these observations may be justified by considering the number of constraints provided by the images of $\mathrm{CP}$ with regard to the size of equation systems involved in the projective factorization and in self-calibration respectively.

\begin{tabular}{|c|c|c|c|c|c|c|c|}
\hline \# views & 3 & 4 & 5 & 6 & 8 & 10 & 12 \\
\hline Error $(\%)$ & 16.0 & 10.1 & 8.8 & 7.5 & 6.8 & 6.1 & 5.1 \\
\hline
\end{tabular}

Table 3: Median of errors on focal length vs. the number of views.

Lastly, Table. 3 shows median errors on the focal length for the proposed method while varying the number of views, considering 20 natural points corrupted by a noise $\sigma=1.0$ pixel. The focal length $f_{k}$ of each projective camera $\mathrm{P}_{k}$ was computed as the least squares solution of the problem $\operatorname{diag}\left(\left(f^{k}\right)^{2},\left(f^{k}\right)^{2}, 1\right) \approx \mathrm{P}_{k} \mathrm{Q}_{\infty}^{*} \mathrm{P}_{k}^{T}$.

\subsection{Real experiments}

The proposed paradigm has been tested on four image sets extracted from video sequences. Table 4 provides information about each experiment. In these experiments, we compared different multiple SfM processing sequences, referred to as $\mathrm{PF}\left[n_{1}\right]+\mathrm{SC}\left[n_{2}\right]$ as in simulation experiments.

Images of $\mathrm{CP}$ were computed based on different primitives (see Table. 4) present in the scene. The "classical" self-calibration algorithm (referred to as SC[0]) was implemented as in [6] from which the linear equations describe with $n_{2} \geq 1$ was adapted in the same way. The dense reconstructions computed by the PMVS software (described in [1]) allow to visually evaluate the performance of the different methods. Selected feature points were extracted and tracked using the iterative KLT algorithm. Inliers, respecting epipolar geometry constraints, were selected using 


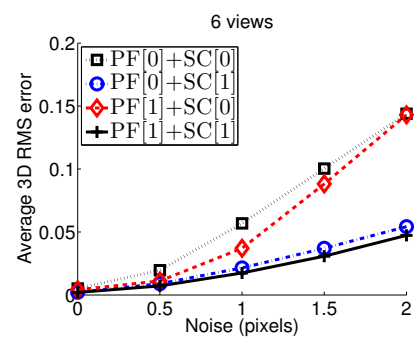

(a)

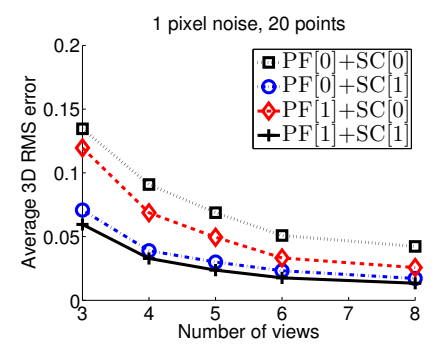

(b)

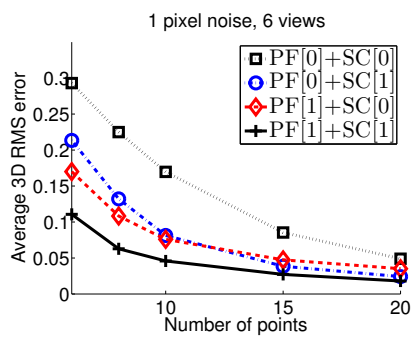

(c)

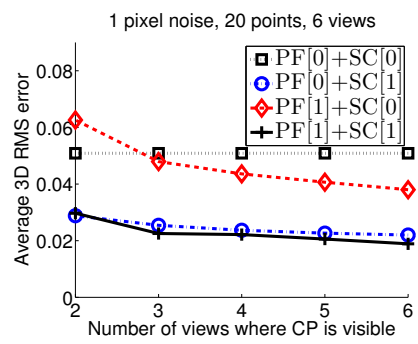

(d)

Figure 3: Averages of the 3D RMS errors (a) vs. the noise applied on point positions (b) $v s$. the number of views (c) vs. the number of views where one CPP is visible (missing ICP) (d) vs. the number of natural points. See text for details.

\begin{tabular}{|c|c|c|c|c|}
\hline Experiment & Ringo & Car & Face & Dragon \\
\hline \multirow{2}{*}{ Camcorder } & Iphone & Nikon & Iphone & Nikon \\
& 4 S (back) & $\mathrm{J} 1$ & 4S (front) & $\mathrm{J} 1$ \\
\hline Image & 1920 & 1920 & 640 & 1920 \\
dimensions & 1080 & 1080 & 480 & 1080 \\
\hline Nb. views & 6 & 8 & 5 & 3 \\
\hline Nb. points & 12 & 7 & 11 & 54 \\
\hline Missing ICP & no & no & no & yes \\
\hline \multirow{2}{*}{ 2D Eucl. Structure } & $\begin{array}{c}\text { concentric } \\
\text { circles }\end{array}$ & $\begin{array}{c}\text { coplanar } \\
\text { circles }\end{array}$ & $\begin{array}{c}\text { parallel } \\
\text { circles }\end{array}$ & grid \\
\hline Euclidean BA & no & no & yes & yes \\
\hline
\end{tabular}

Table 4: Information related to the four experiments. BA means bundle adjustment and points refers to points which are not circular points.

\section{RANSAC algorithm.}

Ringo. In the first experiment, we matched 12 points of a toy figurine seen in 6 views (see Fig. 4 (a)). Images of CP were computed from images of concentric circles lying on a planar marker. The dense reconstruction based on Euclidean cameras computed with $\mathrm{PF}[1]+\mathrm{SC}[0]$ was inconsistent while $\mathrm{PF}[1]+\mathrm{SC}[1]$, including images of $\mathrm{CP}$ in self-calibration, worked properly, providing results shown in Fig. 4 (b).

Car. In this experiment, we matched 7 points of a car seen in 8 views (see Fig. 4(c)). Images of $\mathrm{CP}$ which are the (complex) intersection points of the two coplanar circles formed by the car's wheels are computed as described in [15]. Dense reconstructions based on Euclidean cameras computed with $\mathrm{PF}[0]+\mathrm{SC}[0]$ and $\mathrm{PF}[1]+\mathrm{SC}[0]$ were inconsistent even if the one from $\mathrm{PF}[1]+\mathrm{SC}[0]$ involving images of $\mathrm{CP}$ in the projective factorization is slightly better than the one from $\mathrm{PF}[0]+\mathrm{SC}[0]$ based on only natural points. $\mathrm{PF}[1]+\mathrm{SC}[1]$ performed well, providing results shown Fig. 4(d). We used the result of the proposed method as initial Euclidean reconstruction in an incremental SfM procedure for which results are shown in Fig. 5.

Face. In this experiment, we matched 11 points of a human face seen in 5 views (see Fig. 1(b)) - details are given in the legend). Dense reconstruction based on Euclidean cameras computed with $\mathrm{PF}[1]+\mathrm{SC}[0]$ failed to provide any consistent results where our method worked properly, providing results shown Fig. 1(d).

Dragon. In the last experiment, we matched 54 points of a dragon seen in 3 views (see Fig. 1(a)). Plane-to-image homographies induced by a small grid provided images of $\mathrm{CP}$ in view 1 and 3 . Only the proposed method, starting from the same projective reconstruction, reached to provide good results by adding circular points in self-calibration (see Fig. 1(c)). For further details see legend Fig. 1. We can notice that performing self-calibration without including additional images of $\mathrm{CP}$ can fail when there is a small number of points (see Fig. 4(b), Fig. 4(d), and Fig. 1(d)) but also may sometimes fail with a quite large number of points while the number of views is very low (see Fig. 1(c)). These observations confirm parts of simulation results provided in the paper.

\section{Conclusion}

The purpose of this work was first to show how to introduce images of circular points in a projective factorization algorithm. We dealt with the problem of recovering complex scale factors of images of such features and how to compute missing elements. In a second part, we showed that it is possible to combine "classical" linear self-calibration constraints on the absolute dual quadric, under the assumption that shape pixels and principal points are known, with additional ones derived from the images of the circular points. We described the additional independent equations brought by the absolute line-pair, back-projections of the image of some circular point-pair and how to compute them. Experiments on synthetic and real data validated the proposed algorithms and its performance (codes can be found at the URL ubee.enseeiht.fr/vision/ICCV13).

Assessing the effect of position and orientation of introduced planar shapes on the self-calibration quality in order to optimise it could be investigated. Thus, such new self- 

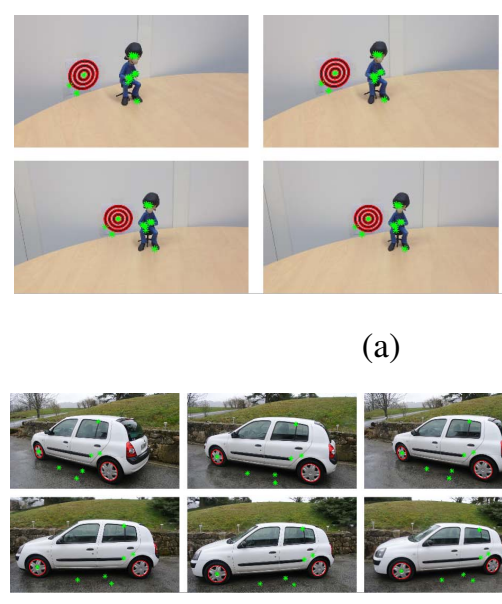

(a)
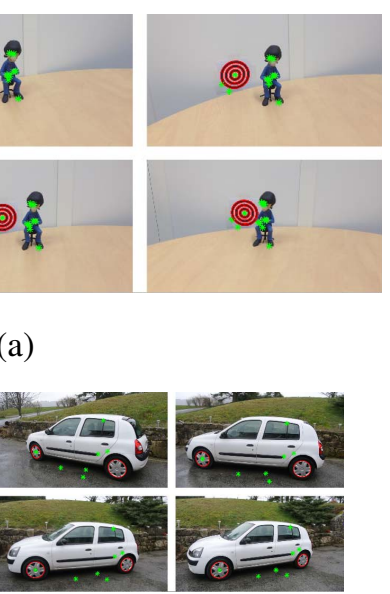

(c)

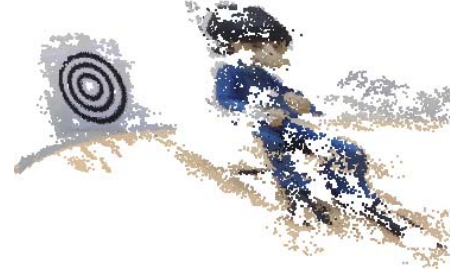

$\mathrm{PF}[1]+\mathrm{SC}[0]$

(b)

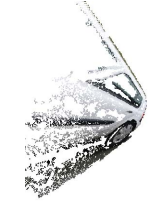

$\mathrm{PF}[0]+\mathrm{SC}[0]$

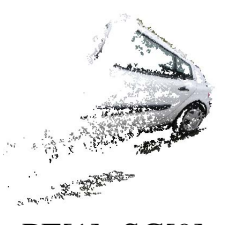

$\mathrm{PF}[1]+\mathrm{SC}[0]$

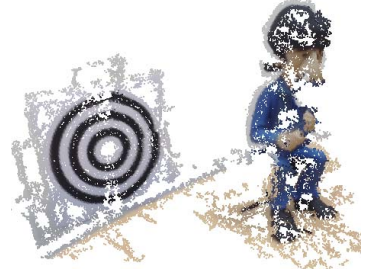

$\mathrm{PF}[1]+\mathrm{SC}[1]$

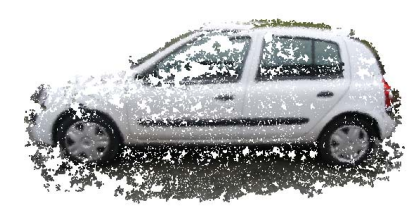

$\mathrm{PF}[1]+\mathrm{SC}[1]$

(d)

Figure 4: Ringo. (a) Input images. (b) Dense reconstructions based on Euclidean cameras computed with $\mathrm{PF}[1]+\mathrm{SC}[0]$ at left and with $\mathrm{PF}[1]+\mathrm{SC}[1]$ at right. See text for details. Car. (a) Input images. (b) Dense reconstructions based on Euclidean cameras computed with $\mathrm{PF}[0]+\mathrm{SC}[0], \mathrm{PF}[1]+\mathrm{SC}[0]$ and $\mathrm{PF}[1]+\mathrm{SC}[1]$ from left to right. See text for details.

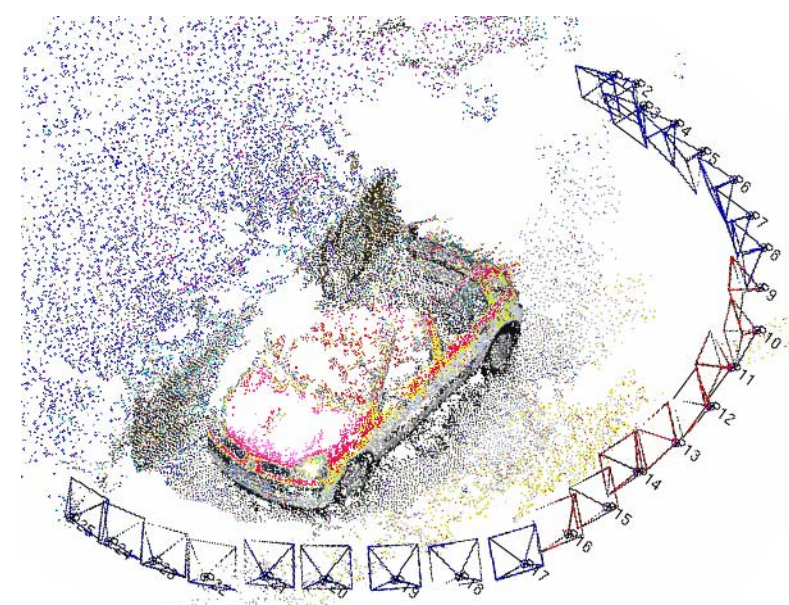

Figure 5: Car. Using the whole Car image sequence, from which the 8 images of Fig. 4(b) were selected, the indermediary camera poses were obtained by incremental resection and all the results were refined by bundle adjustment.

calibration devices could be used in some application, e.g. camera tracking, to better control the self-calibration quality and ensuring the presence of a reliable subset of key-frames to upgrade the projective SfM to Euclidean. In practice, such approach would offer a very good trade-off between flexibility and performance, especially when classical approaches fail.

\section{References}

[1] Y. Furukawa and J. Ponce. Accurate, Dense, and Robust Multiview Stereopsis. PAMI, 32(8), 2010.

[2] P. Gurdjos, A. Bartoli, and P. Sturm. Is dual linear self- calibration artificially ambiguous? ICCV, 2009.

[3] R. I. Hartley and A. Zisserman. Multiple View Geometry in Computer Vision. Cambridge University Press, second edition, 2004.

[4] J. Oliensis and R. Hartley. Iterative Extensions of the Sturm/Triggs Algorithm: Convergence and Nonconvergence. PAMI, 29(12), 2007.

[5] M. Pollefeys, R. Koch, and L. J. Van Gool. Self-Calibration and Metric Reconstruction Inspite of Varying and Unknown Intrinsic Camera Parameters. IJCV, 32(1), 1999.

[6] M. Pollefeys, L. J. Van Gool, M. Vergauwen, F. Verbiest, K. Cornelis, J. Tops, and R. Koch. Visual Modeling with a Hand-Held Camera. IJCV, 59(3), 2004.

[7] J. Ponce, K. McHenry, T. Papadopoulo, M. Teillaud, and B. Triggs. On the Absolute Quadratic Complex and Its Application to Autocalibration. CVPR, 2005.

[8] V. Rabaud. Vincent's Structure from Motion Toolbox. http: //vision.ucsd.edu/ vrabaud/toolbox/.

[9] J. I. Ronda, A. Valdés, and G. Gallego. Line Geometry and Camera Autocalibration. JMIV, 32(2), 2008.

[10] Y. Seo and A. Heyden. Auto-calibration by linear iteration using the DAC equation. IVC, 22(11), 2004.

[11] P. Sturm and S. J. Maybank. On Plane-Based Camera Calibration: A General Algorithm, Singularities, Applications. CVPR, 1999.

[12] P. Sturm and B. Triggs. A Factorization Based Algorithm for Multi-Image Projective Structure and Motion. ECCV, 1996.

[13] B. Triggs. Autocalibration and the absolute quadric. CVPR, 1997.

[14] A. Valdés and J. I. Ronda. Camera Autocalibration and the Calibration Pencil. JMIV, 23(2), 2005.

[15] Y. Wu, X. Li, F. Wu, and Z. Hu. Coplanar circles, quasiaffine invariance and calibration. IVC, 24(4), 2006.

[16] Z. Zhang. A Flexible New Technique for Camera Calibration. PAMI, 22(11), 2000. 